\title{
Periodic Pattern Formation Analysis Numerically in a Chemical Reaction-Diffusion System
}

\author{
A. K. M. Nazimuddin ${ }^{\text {a, }}{ }^{*}$, Md. Showkat Ali ${ }^{\text {b }}$ \\ ${ }^{a}$ Department of Mathematical and Physical Sciences, East West University, Dhaka-1212, Bangladesh. \\ ${ }^{b}$ Department of Applied Mathematics, University of Dhaka, Dhaka-1000, Bangladesh.
}

Received: 11 February 2019; Accepted: 06 March 2019; Published: 08 July 2019

\begin{abstract}
In this paper, we analyze the pattern formation in a chemical reaction-diffusion Brusselator model. Twocomponent Brusselator model in two spatial dimensions is studied numerically through direct partial differential equation simulation and we find a periodic pattern. In order to understand the periodic pattern, it is important to investigate our model in one-dimensional space. However, direct partial differential equation simulation in one dimension of the model is performed and we get periodic traveling wave solutions of the model. Then, the local dynamics of the model is investigated to show the existence of the limit cycle solutions. After that, we establish the existence of periodic traveling wave solutions of the model through the continuation method and finally, we get a good consistency among the results.
\end{abstract}

Index Terms: Reaction Diffusion System, Periodic Traveling wave, Pattern Formation, Brusselator Model.

(C) 2019 Published by MECS Publisher. Selection and/or peer review under responsibility of the Research Association of Modern Education and Computer Science

\section{Introduction}

Pattern formation of a nonlinear reaction-diffusion (R-D) system of partial differential equations (PDEs) can be described by the Periodic traveling wave (PTW) solutions of such system. In paper [1], authors first studied the PTW solutions of a coupled oscillatory R-D system. The PTW solutions have also been noticed in ecological $[2,3,4]$, biological $[5,6]$, physical $[7,8,9]$ and chemical $[10,11,12]$ systems. Method of continuation is a powerful method to study the PTW solutions of a R-D system [13]. In this paper, we consider an activator-inhibitor Brusselator model which represents an autocatalytic oscillating chemical reaction and the pattern formations for this model are discussed in [14, 15, 16, 17]. In paper [18], author theoretically proved the existence of the Hopf bifurcation of the Brusselator model which reveals the existence of PTW solutions of

* Corresponding author.

E-mail address: nazimuddin@ewubd.edu 
the model. In paper [19], authors discussed the asymptotic behavior of the solutions of the Brusselator model numerically and focused on the stable pattern formation. In paper [20], author studied various types of pattern formation of the Brusselator model arising in chemical reactions with the numerical investigation. We are interested to establish the existence of PTW solutions of the Brusselator model numerically as a function of wave speed and a bifurcation parameter $b$ throughout the periodic pattern formation.

Our goal of this present paper is to study the periodic pattern formation of the model (1). We obtain spatiotemporal periodic pattern by using the direct PDE numerical computations in two dimensions. Numerical simulations in one dimension are also performed to show the existence of PTW solutions of (1). We prove the existence of Hopf bifurcation point of the system (2) numerically, by using the parameter $b$ as a bifurcation parameter. We establish the existence of the PTW solutions as a function of the parameter $b$ in the model (1) via the method of continuation.

The remainder part of this paper is organized as follows. In section 2, we describe a nonlinear R-D system for the standard Brusselator model. In subsection 3.1, periodic pattern formation through the direct numerical PDE simulation in two dimensions is discussed. In subsection 3.2, we show the PTW solutions of the model through PDE numerical simulation in one dimension. Local dynamics analysis is discussed in subsection 3.3. In subsection 3.4, we establish the existence of PTW solutions of the model using the continuation method. Finally, some conclusions are given in section 4.

\section{Model}

The R-D system of equations in two variables for the standard Brusselator model is given by [21]:

$$
\begin{aligned}
& \frac{\partial u}{\partial t}=D_{u} \Delta u+a-(b+1) u+u^{2} v, \\
& \frac{\partial v}{\partial t}=D_{v} \Delta v+b u-u^{2} v,
\end{aligned}
$$

where $u$ and $v$ are the dimensionless concentration called activator and inhibitor and whose corresponding diffusion coefficients are $D_{u}$ and $D_{v}$ respectively. The reaction kinetics are $f(u, v)=a-(b+1) u+u^{2} v$ and $g(u, v)=b u-u^{2} v$ for the activator and inhibitor respectively where ${ }^{a}$ and $b$ are the kinetic parameters. The equilibrium for the system $f(u, v)=0$ and $g(u, v)=0$ is $(a, b / a)$.

\section{Results and Discussions}

\subsection{Periodic Patten Formation in the Two Dimensional Spaces through PDE Simulation}

In this section, we use alternating direction implicit (ADI) method with Neumann boundary conditions to perform a series of direct PDE numerical simulations of (1) in two dimensions. Numerical simulation is performed on the spatiotemporal grid $\left(x_{i}, y_{j}\right)$ with $x_{i}=i \Delta x, i=0, \cdots, N_{x}$ and $y_{j}=j \Delta y, j=0, \cdots, N_{y}$ where $\Delta x=\Delta y$ for a uniform mesh grid and time $t_{n}=n \Delta t, n=0,1,2,3 \cdots$, where $\Delta t$ is the time step. Therefore, the space steps in the $x$-direction and in the $y$-direction are as follows: 


$$
\Delta x=\frac{L_{x}}{N_{x}}, \Delta y=\frac{L_{y}}{N_{y}}, N_{x}, N_{y} \in \mathbb{Z},
$$

where $0<x<L_{x}$ and $0<y<L_{y}$ is used as the domain in the $(x, y)$ parameter plane. In (1), we represent the grid approximations by $U_{i, j}^{n} \approx u\left(x_{i}, y_{j}, t_{n}\right)$ and $V_{i, j}^{n} \approx v\left(x_{i}, y_{j}, t_{n}\right)$. Therefore, the full discrete grid approximation of $U_{i, j}^{n}$ is as follows:

$$
\begin{aligned}
& \frac{U_{i, j}^{n+1 / 2}-U_{i, j}^{n}}{\Delta t / 2}=D_{u} \frac{U_{i-1, j}^{n+1 / 2}-2 U_{i, j}^{n+1 / 2}+U_{i+1, j}^{n+1 / 2}}{\Delta x^{2}}+D_{u} \frac{U_{i, j-1}^{n}-2 U_{i, j}^{n}+U_{i, j+1}^{n}}{\Delta y^{2}}+f\left(U_{i, j}^{n}, V_{i, j}^{n}\right) \\
& \frac{U_{i, j}^{n+1}-U_{i, j}^{n+1 / 2}}{\Delta t / 2}=D_{u} \frac{U_{i-1, j}^{n+1 / 2}-2 U_{i, j}^{n+1 / 2}+U_{i+1, j}^{n+1 / 2}}{\Delta x^{2}}+D_{u} \frac{U_{i, j-1}^{n+1}-2 U_{i, j}^{n+1}+U_{i, j+1}^{n+1}}{\Delta y^{2}}+f\left(U_{i, j}^{n+1 / 2}, V_{i, j}^{n+1 / 2}\right)
\end{aligned}
$$

Equation (3) indicates the first half of the total time step and (4) indicates the rest half of the total time step. The central difference operator is defined as $\delta_{x} U_{i, j}^{k}=U_{i+1 / 2, j}^{k}-U_{i-1 / 2, j}^{k}$ and a similar formula can be defined for $\delta_{y}$. Equialently, we can define the approximation equations for $V_{i, j}^{n}$.

Table 1. Typical parameter values of (1) for the numerical computations.

\begin{tabular}{lcccc}
\hline Parameters & $a$ & $b$ & $D_{u}$ & $D_{v}$ \\
\hline Values & 3.0 & 14.0 & 3.0 & 10.0 \\
\hline
\end{tabular}

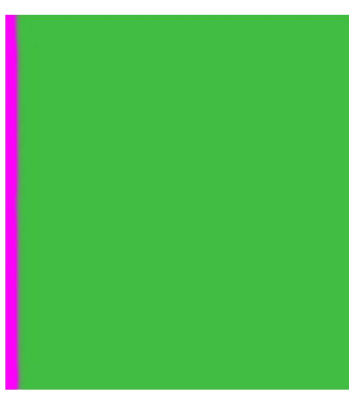

(a)

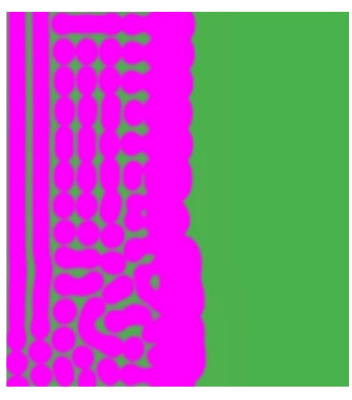

(b)

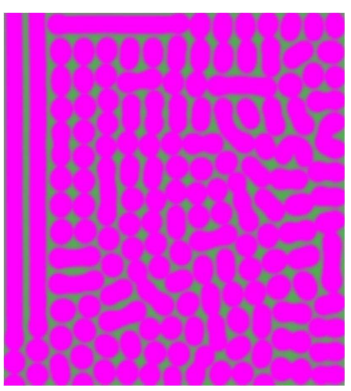

(c)

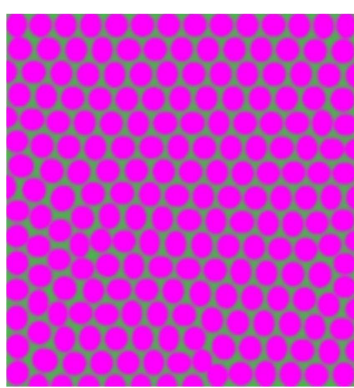

(d)

Fig.1. Pattern evolution as a function of time. (a)at $t=0$ (b) at $t=47$ (c) at $t=122$ (d) at $t=2000$.

In this simulation, we use $\Delta x=\Delta y=0.5$ as space step and $\Delta t=0.04$ as time step on a grid of $220 \times 220$ elements and eventually, we get a periodic spot pattern. Again, it was checked that the decreasing values of step size did not lead to any changes in the results. We continue our numerical simulations until they are in a stationary or until they have behavior that the characteristics results do not seem anymore. Fig.1 shows the dynamics of a periodic pattern of (1) as a function of time. Here, we consider a small perturbation of the steady state solution as an initial guess and continue our simulation process for a long time until we get a periodic pattern. We use the parameter values of (1) as mentioned in Table 1. Fig.1(a) shows the initial data at time 
$t=0$. Fig.1(b), Fig.1(c) shows the development process of the spot pattern at time $t=47$ and $t=122$ respectively. Finally, we get a periodic spot pattern at time $t=2000$ which shows in Fig.1(d).

\subsection{Existence of PTW Solutions in the One Dimensional Space through PDE Simulation}

In this subsection, we compare the results of (1) obtained in the previous subsection 3.1 with the corresponding direct PDE numerical simulations of (1) in one dimensional space. Fig.2 shows the qualitative behavior of the PTW solutions of (1) with the periodic patterns.

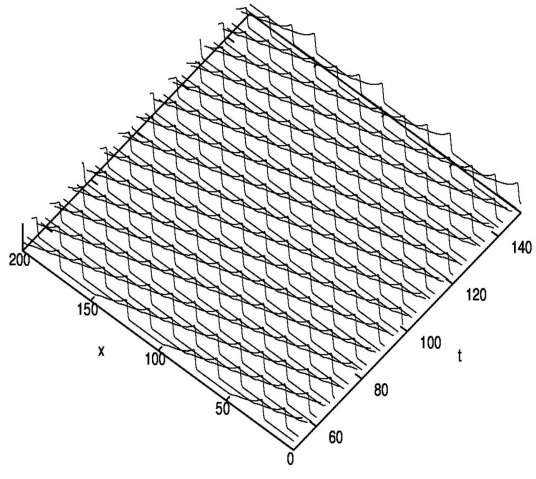

(a)

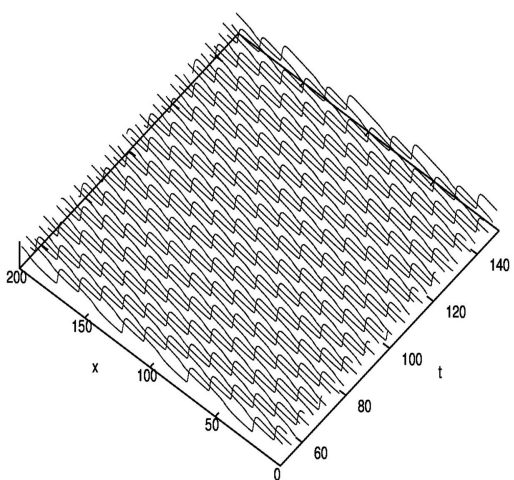

(b)

Fig.2. Space-time plot via direct PDE simulation. (a) Solutions of activator, ${ }^{u}$ (b) Solutions of inhibitor, $v$.

Fig.2(a) and Fig.2(b) shows that the PTW solutions of the activator, $u$ and the inhibitor, $v$ respectively. We apply an implicit scheme with periodic boundary conditions over the domain $\left[0, D_{x}\right]$. Here $D_{x}$ represents the system size which is defined by $D_{x}=n \times p$ where $n$ is the number of pulses and $p$ is the spatial period. We consider, $d x=0.09$ as space size and $d t=0.01$ as time step on 2181 grid elements and other parameter settings remain the same as those in Fig.1. Here, we consider a small perturbation of the steady state solution as the initial data and continue our simulation process for a long time until we get a stable pattern. In this simulation, we take $D_{x}=200$ as the system size with four pulses that means, the spatial period is $p=50$ and also we take $50 \leq t \leq 150$ as the time range for the solutions of (1). Finally, we obtain periodic pattern solutions of the activator, $u$ as well as the inhibitor, $v$. Hence, we get a good agreement between the results obtained from this subsection and the result from the subsection 3.1 .

\subsection{Existence of Limit Cycles (Local Dynamics Analysis)}

In this subsection, we establish the existence of limit cycle solutions of the local dynamics of the model (1). Local dynamics of the model (1) without diffusion term can be represented by the following system of ordinary differential equations (ODEs). 


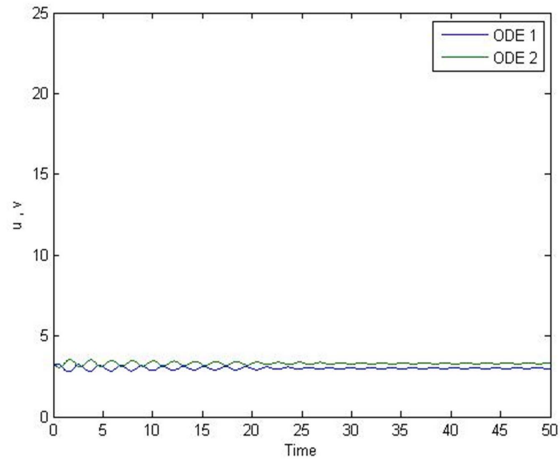

(a)

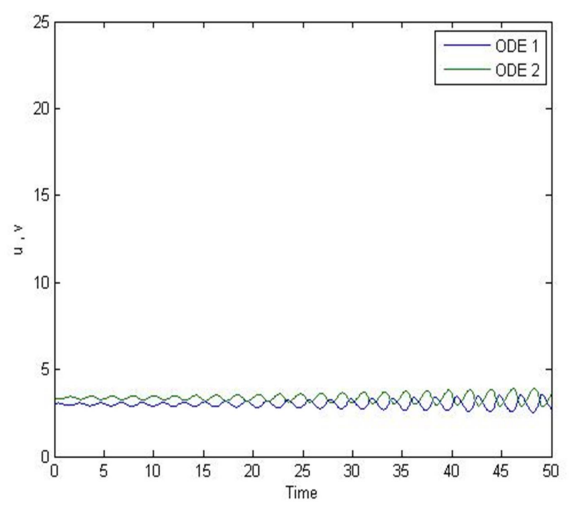

(c)

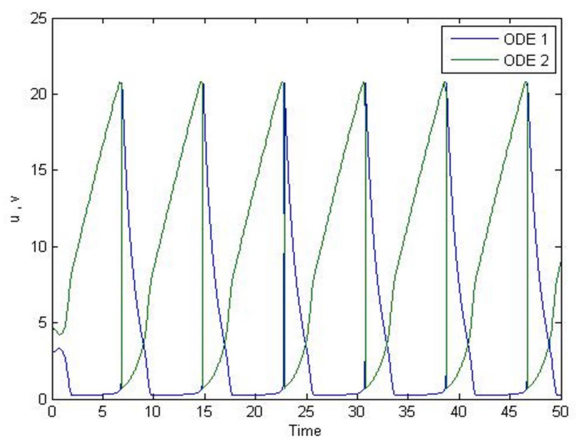

(e)

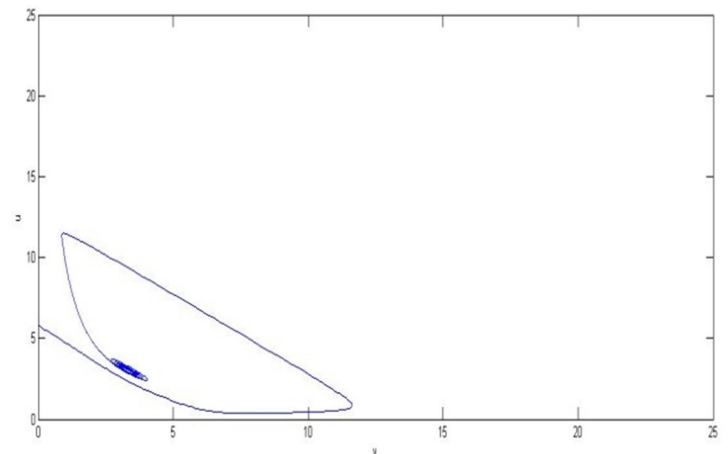

(b)

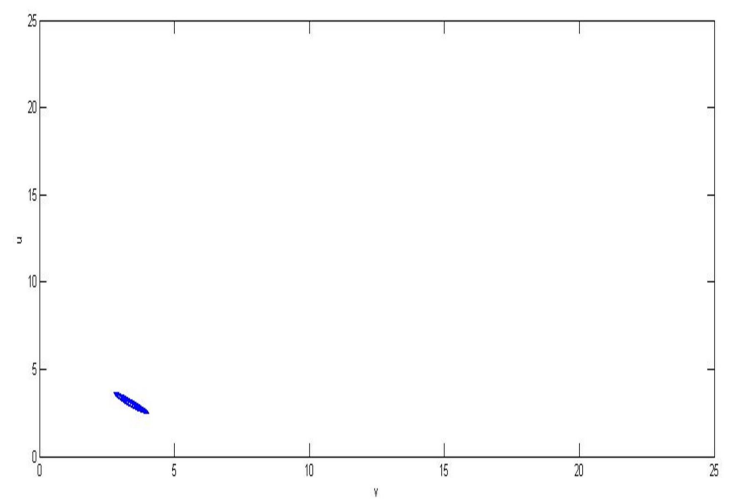

(d)

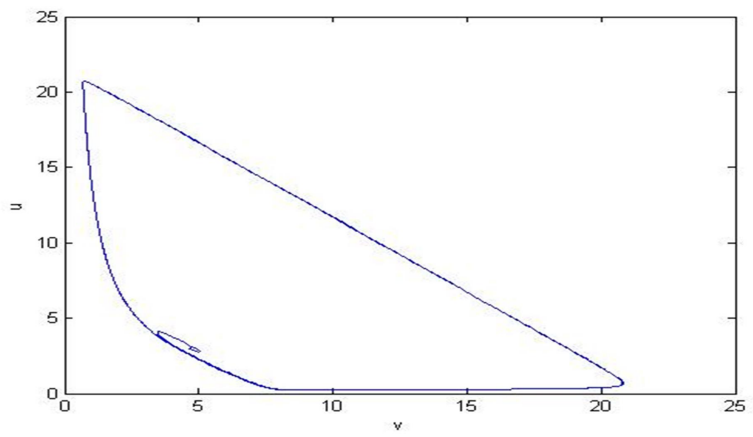

(f)

Fig.3. An illustration of the local dynamics of the model (1) which is represented by (5).

$$
\begin{aligned}
& \frac{d u}{d t}=a-(b+1) u+u^{2} v \\
& \frac{d v}{d t}=b u-u^{2} v .
\end{aligned}
$$


A periodic solution $(u, v)$ is a periodic orbit or limit cycle of the system of ODEs (5). Linear stability analysis shows that when $b<1+a^{2}$ then the equilibrium point $(a, b / a)$ is stable and all other non-equilibrium solutions of (5) approach to the unstable limit cycle. Also, when $b>1+a^{2}$ then the equilibrium point $(a, b / a)$ is unstable and all other non-equilibrium solutions of (5) approach to the stable limit cycle. Hence, we get an equation for the Hopf bifurcation points and which is $b=1+a^{2}$. Now, we investigate the system of ODEs (5) numerically to verify the linear stability analysis results.

In Fig.3, we plot activator and inhibitor densities $u$ and $v$ with respect to time as well as $u$ versus $v$ where we take $a=3.0$. In this case, the kinetics have a Hopf bifurcation at $b=10.0$ and we get a stable limit cycle solution for greater values of $b=10.0$. Fig.3(a) and Fig.3(b) represents the solutions of (5) when $a=3.0$ and $b=9.9$, where we get the stable equilibrium point $(3,3.3)$ and shows the existence of the unstable limit cycle. Fig.3(c) and Fig.3(d) represents the solutions of (5) when $a=3.0$ and $b=10.1$ where we get the unstable equilibrium point $(3,3.37)$ shows the existence of the stable limit cycle. Fig.3(e) and Fig.3(f) represents the solutions of (5) when $a=3.0$ and $b=14$, where we get the unstable equilibrium point $(3,4.67)$ and shows the existence of the stable limit cycle. From the stable limit cycle solutions of (5), we see that the cycles are of low amplitude for $b$ close to the Hopf bifurcation value $b=10.0$ and they increase in amplitude as $b$ is increased.

\subsection{Existence of PTW Solutions of the Model via Continuation Method}

In order to provide the instability of a R-D system, it is important to investigate the PTW solutions of (1) via traveling wave coordinate. In this section, we use a software package WAVETRAIN [13] which is based on the continuation method and we establish the existence of PTW solutions of (1) in the two dimensional parameter plane. Traveling wave coordinate $z=x-c t$ is used in our calculation where ${ }^{c}$ is the wave speed and also $x$ and $t$ are the space and time coordinates, respectively. By putting $u(x, t)=U(z)$ and $v(x, t)=V(z)$ in $(1)$, we get the following traveling wave equations.

$$
\begin{aligned}
& D_{u} \frac{d^{2} U}{d z^{2}}+c \frac{d U}{d z}+a-(b+1) U+U^{2} V=0, \\
& D_{v} \frac{d^{2} V}{d z^{2}}+c \frac{d V}{d z}+b U-U^{2} V=0 .
\end{aligned}
$$

Now we rewrite the system of equations (6) as a system of four dimensional ODEs as follows:

$$
\begin{aligned}
& \frac{d U}{d z}=P, \\
& \frac{d P}{d z}=\left(-c P-a+(b+1) U-U^{2} V\right) / D_{u}, \\
& \frac{d V}{d z}=Q, \\
& \frac{d Q}{d z}=\left(-c Q-b U+U^{2} V\right) / D_{v} .
\end{aligned}
$$




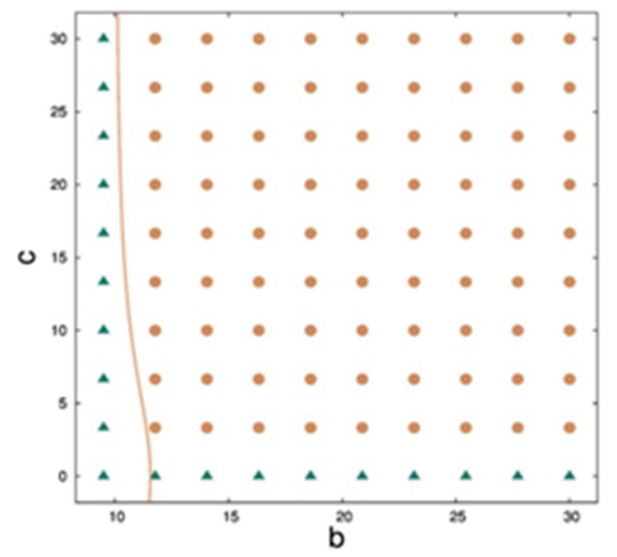

Fig.4. Existence of periodic traveling wave solutions of (1) as a function of parameter $b$ and the wave speed $c$.

A PTW solution $(U(z), V(z))$ represents the existence of periodic orbit or limit cycle solution of the system (7) via Hopf bifurcation theorem $[22,23]$ as a bifurcation parameter passes through the critical value. In simple cases, the branch of the solution (7) has at least one terminating at a Hopf bifurcation point [13] and in complex problems, the solution branch starts and ends at homoclinic solution. A periodic traveling wave solution from the simulation of the partial differential equation is required as an initial solution for the continuation. The parameter $b$ is considered as the bifurcation parameter and the other parameter values of (1) are the same as in Fig.1. Fig. 4 represents the existence of the PTW solutions on a grid of $10 \times 10$ elements. In the parameter plane, the green triangle represents that periodic traveling wave solution does not exist and also the orange line represents that the locus of Hopf bifurcation points with respect to the wave speed. Also, the brown circle indicates that the existence of the PTW solution corresponding to the wave speed ${ }^{c}$ and the parameter value $b$.

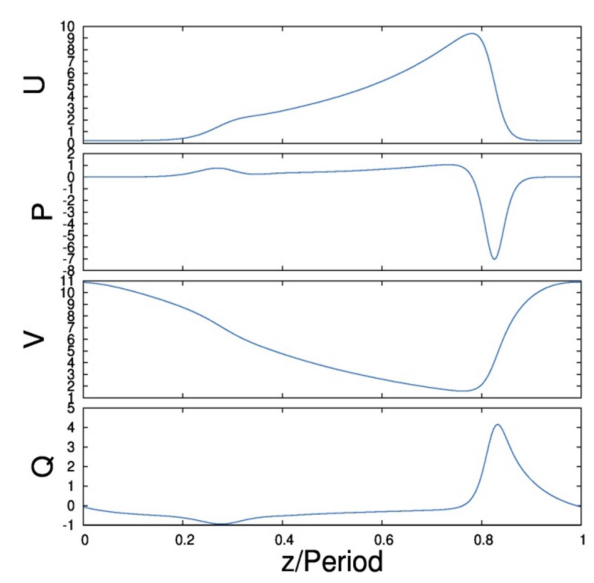

(a)

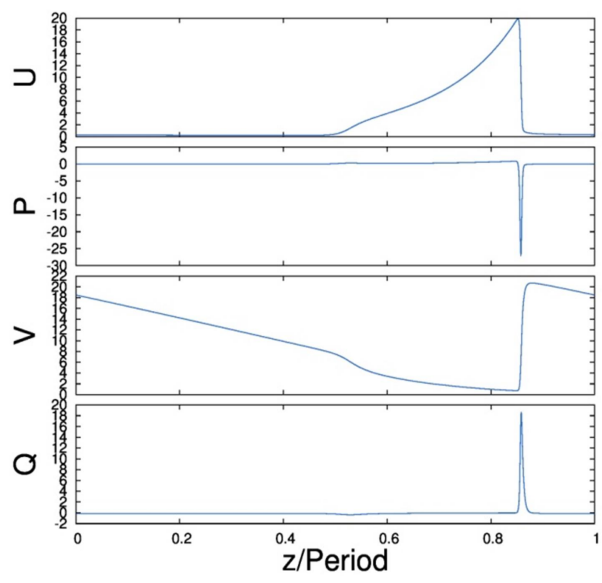

(b)

Fig.5. Two PTW solution profiles of (1) as a function of the parameter $b$ and wave speed $c$. 
Fig.5 shows two different PTW solution profiles of (1) as a function of control parameter $b$ and wave speed $c$. Here we calculate solution profiles for $b=14.0$ with different wave speeds and the other parameter values of our model (1) are $a=3.0, D_{u}=3.0$ and $D_{v}=10.0$. In Fig.5, symbols $U, P, V$ and $Q$ are the internal names of the state variables of (1). According to the $(b, c)$ parameter plane in Fig.4, Fig.5(a) represents the PTW solution profile for the bifurcation parameter value $b=14.0$ with the wave speed $c=5.0$ and the calculated period of the wave is 26.78. Also, Fig.5(b) represents the PTW solution profile for the bifurcation parameter value $b=14.0$ with the wave speed $c=20.0$ and the calculated period of the wave is 158.97 . These figures in Fig.5 indicate that increasing the value of wave speed $c$ increases the periods of the PTW solutions of (1) at fixed bifurcation parameter value. However, the periods are not changing quickly as wave speed $c$ changes. In paper [1], authors discussed the PTW solutions of the R-D system and the stability of the waves based on the periods of the corresponding waves. They showed that waves a sufficiently small period are always unstable whereas those with a sufficiently large period are always stable. Our Fig.5(a) shows the small period 26.78 with the small wave speed $c=5.0$ and the figure looks like an unstable PTW solution and similarly Fig.5(b) shows the large period 158.97 corresponding to the high wave speed $c=20.0$ and the figure looks like a stable PTW solution.

\section{Conclusion}

We showed the periodic pattern dynamics of a non-linear R-D Brusselator model by the direct numerical PDE simulation in two dimensions. In the numerical simulation process, we figured out a periodic spot pattern. Besides, we studied the existence of PTW solutions numerically in one dimensional space. We showed the existence of periodic solutions as well as limit cycles of the local dynamics of our model. We also studied the existence of PTW solutions in the parameter plane as a function of one parameter family via continuation method. Moreover, we showed several PTW solution profiles for $b=14.0$ with different wave speeds corresponding to the continuation result. Thus, we found a consistent result between the continuation result and the result from the direct PDE simulations.

\section{References}

[1] Kopell, N. and Howard, LN. (1973). Plane-wave solutions to reaction-diffusion equations. Stud Appl. Math, 52:291-328.

[2] Ranta, E. and Kaitala, V. (1997). Travelling waves in vole population dynamics, Geochim. Cosmochim. Acta, 61:3503-3512.

[3] Bierman, S. M., Fairbairn, J. P., Petty, S. J., Elston, D. A., Tidhar, D. and Lambin, X. (2006). Changes over time in the spatiotemporal dynamics of cyclic populations of field voles (Microtus agrestis L.), The American Naturalist, 167 (4):583-590.

[4] Sherratt, J. A., Smith, M. J. (2008). Periodic travelling waves in cyclic populations: field studies and reaction-diffusion models, Journal of the Royal Society Interface, 5 (22):483-505.

[5] DeVille, R. E. L. and Eijnden, E. V. (2007). Wavetrain response of an excitable medium to local stochastic forcing, Nonlinearity, 20 (1):51-74.

[6] Sherratt, J. A., Lord, G. J. (2007). Nonlinear dynamics and pattern bifurcations in a model for vegetation stripes in semi-arid environments, Theoretical Population Biology, 71 (1):1-11.

[7] Steinberg, V., Fineberg, J., Moses, E. and Rehberg, I. (1989). Pattern selection and transition to turbulence in propagating waves, Physica D: Nonlinear Phenomena, 37:359-383.

[8] Van Hecke, M., (2003). Coherent and incoherent structures in systems described by the 1D CGLE: experiments and identification, Physica D: Nonlinear Phenomena, 174 (1):134-151. 
[9] Van Saarloos, W. (2003). Front propagation into unstable states, Physics Reports, 386 (2): $29-222$.

[10] Epstein, I. R. and Showalter, K. (1996). Nonlinear chemical dynamics: oscillations, patterns, and chaos, The Journal of Physical Chemistry, 100(31):13132-13147.

[11] Vanag, V. K., Epstein, I. R. (2008). Design and control of patterns in reaction-diffusion systems, Chaos: An Interdisciplinary Journal of Nonlinear Science, 18 (2):(026107)1-11.

[12] Bordyugov, G., Fischer, N., Engel, H., Manz, N. and Steinbock, O. (2010). Anomalous dispersion in the Belousov-Zhabotinsky reaction: Experiments and modeling, Physica D: Nonlinear Phenomena, 239 (11): 766-775.

[13] Sherratt, J. A. (2012). Numerical continuation methods for studying periodic travelling wave (wavetrain) solutions of partial differential equations, Applied Mathematics \& Computation, 218(9): 4684-4694.

[14] Dutt, AK. (2010). Turing pattern amplitude equation for a model glycolytic reaction-diffusion system, Journal of mathematical chemistry, 48(4):841-855.

[15] Kolokolnikov, T., Erneux, T., and Wei, J. (2006). Mesa-type patterns in the one-dimensional brusselator and their stability, Physica D: Nonlinear Phenomena, 214(1):63-77.

[16] Tzou, JC., Matkowsky, B. J. and Volpert, V. A. (2009). Interaction of turing and hopf modes in the super diffusive brusselator model, Applied Mathematics Letters, 22(9):1432-1437.

[17] Yang, L., Zhabotinsky, A. M., and Epstein, I. R. (2004). Stable squares and other oscillatory turing patterns in a reaction-diffusion model, Physical review letters, 92(19):198-303.

[18] Feng, B. (1988). Periodic Travelling-wave Solution of Bruselator, Acta Math. Appl. Sinica, 4(4): $324-332$.

[19] Anguelov, R. \& Stoltz, S. M. (2017). Stationary and oscillatory patterns in a coupled Brusselator model, Mathematics and Computers in Simulation, 133(C): 39-46.

[20] Alqahtani, AM. (2018). Numerical simulation to study the pattern formation of reaction-diffusion Brusselator model arising in triple collision and enzymatic, Journal of Mathematical Chemistry, 56(6):1543-1566.

[21] Prigogine, I., Lefever, R. (1968). Symmetry breaking instabilities in dissipative systems, The Journal of Chemical Physics, 48(4): 1695-1700.

[22] Hassard, B. D., Kazarinoff, N. D. and Wan, Y. H. (1981). Theory and Applications of Hopf Bifurcation, Cambridge University Press.

[23] Marsden, J. E. and McCracken, M. (1976). The Hopf Bifurcation and its Applications, Applied Math. Series, 19, New-York, Springer-Verlag.

\section{Authors' Profiles}

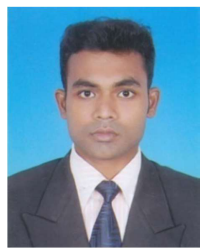

A. K. M. Nazimuddin received his B. S. (Hons) degree in Mathematics and M. S. in Applied Mathematics from the University of Dhaka. He is working as a Senior Lecturer in the Department of Mathematical and Physical Sciences, East West University, Dhaka, Bangladesh. His research interest is on Dynamical Systems, Riemannian Geometry, Symplectic Geometry and Contact Geometry.

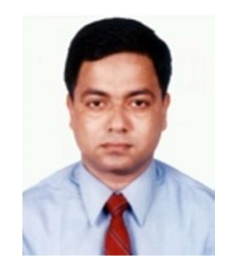

Md. Showkat Ali received his $\mathrm{PhD}$ from the University of Glasgow, Glasgow, UK.. He received his B. S. (Hons) degree in Mathematics and M. S. in Applied Mathematics from the University of Dhaka, Bangladesh. He is working as a Professor in the Department of Applied Mathematics, University of Dhaka. His research interest is on Differential Geometry and Dynamical Systems. He has published about fifty research articles in this field in various reputed national and international journals. 
How to cite this paper: A. K. M. Nazimuddin, Md. Showkat Ali," Periodic Pattern Formation Analysis Numerically in a Chemical Reaction-Diffusion System ", International Journal of Mathematical Sciences and Computing(IJMSC), Vol.5, No.3, pp.17-26, 2019. DOI: 10.5815/ijmsc.2019.03.02 